\title{
Zero Modes on Linked Strings
}

\author{
Jaume Garriga \\ IFAE, Edifici C, Universitat Autònoma de Barcelona \\ E-08193 Bellaterra, Spain. \\ Tanmay Vachaspati 4 \\ Isaac Newton Institute, 20 Clarkson Road, \\ University of Cambridge, Cambridge, CB3 OEH, U.K.
}

\begin{abstract}
We study linked loops of string in the presence of bosonic condensates and fermionic zero modes on the strings. We find that the strings necessarily carry a current if the bosons have an AharanovBohm interaction with the string. The fermionic case is analyzed in the context of the standard model where there are lepton and quark zero modes on $Z$-strings. Here we find that the fermionic ground state in the linked string background is lower than the ground state when the loops are unlinked but otherwise identical. As in the bosonic case, the $Z$-strings carry a non-vanishing electric current in the ground state. The baryon number of the linked configuration is found to agree with previous indirect results. We also evaluate the angular momentum, electromagnetic charge and baryonic three current on the linked $Z$-string configuration. Finally we point out a possible gravitational analogue of the linked string system.
\end{abstract}

\footnotetext{
${ }^{1}$ Address from 1 January 1995: Department of Physics, Case Western Reserve University, Cleveland, OH 44106, USA.
} 


\section{Introduction}

The study of particle-soliton interactions over the last 20 years has led to the discovery of novel physical phenomena (for a few such examples, see [1, 2, 3]). Some of these phenomena have been successfully tested in condensed matter systems where solitons abound and the ideas have also been widely applied to hypothetical particle physics situations. These beautiful ideas are also relevant to the standard model of particle physics even though the model does not contain any topological defects. This is because the ideas have applicability even when the solitons are not truly topological and there are a number of such "non-topological" solitons present in the standard model. In this paper we shall apply some of these ideas to the electroweak $Z$-string - a flux tube containing magnetic flux of the $Z$ boson 4 , 5, 6].

Several facets of the $Z$-string have been studied over the past few years and recently the attention has turned to their interactions with the particles in the standard model. Earnshaw and Perkins [7] have found the quark and lepton zero modes on a straight $Z$-string. This means that $Z$-strings are superconducting and can carry of order $10^{8}$ Amperes of electric current. In another paper it was shown that linked loops of $Z$-string carry baryon number [8]. This result was derived by integrating out the anomaly equation for the baryonic current and did not explicitly consider the fermions interacting with the string. The moment seems ripe for a more direct computation of the baryon number and other charges on linked loops of $Z$-string. This is the underlying motivation for the present work.

The technical analysis given in this paper is inspired by earlier work in which the spectral flow in non-trivial backgrounds was studied. In particular, the paper by Manton on Schwinger electrodynamics in $1+1$ dimensions[9] was immensely helpful as the situation there closely resembles the problem at hand.

The plan of the paper is as follows. In section 2, we set up our conventions, describe electroweak $Z$-strings and recapitulate known results on fermionic zero modes. In section 3 we describe two cases in which the Dirac equation for the fermionic zero modes can be solved completely in terms of the string profile functions. In both cases we need to assume the Bogomolnyi limit [10] in which the masses of the scalar and vector fields making up the string are equal. In the first of the two cases, the fermion is massless (such as the neutrino) while in the second case we have a "super-Bogomolnyi" 
limit in which the fermion mass is equal to the scalar and vector masses and the $Z$-charge on the left-handed fermion vanishes. Then, in section 4 , we describe what happens to bosonic zero modes on linked strings as this is the simplest situation. The main results of this paper are found in section 5 , where we discuss lepton and quark zero modes on linked loops of electroweak $Z$-string. Here we evaluate the energy, angular momentum and the electromagnetic and baryonic currents on the strings. One outcome of this evaluation is that the ground state energy of fermions on linked strings is lower than that on unlinked strings. We also confirm previous findings that linked loops of $Z$-string carry baryon number $2 N_{F} \cos \left(2 \theta_{W}\right)$ where $N_{F}$ is the number of fermion families and $\theta_{W}$ is the Weinberg angle. In addition, we show that the linked strings necessarily carry electromagnetic currents in their ground state. We extend our analysis to loops with linkage greater than one and plot the energy as a function of linkage. We conclude in section 6 and outline a gravitational situation that resembles the system of linked strings and which may have physics similar to that discussed in this paper.

\section{Review}

The bosonic sector of the standard model of the electroweak interactions describes an $S U(2) \times U(1)$ invariant theory with a scalar field $\Phi$ in the fundamental representation of $S U(2)$. It is described by the Lagrangian:

$$
L_{b}=L_{W}+L_{Y}+L_{\Phi}-V(\Phi)
$$

B where,

$$
\begin{gathered}
L_{W}=-\frac{1}{4} W_{\mu \nu a} W^{\mu \nu a} \\
L_{Y}=-\frac{1}{4} Y_{\mu \nu} Y^{\mu \nu}
\end{gathered}
$$

where $W_{\mu \nu}^{a}$ and $Y_{\mu \nu}$ are the field strengths for the $S U(2)$ and $U(1)$ gauge fields $W_{\mu}^{a}$ and $Y_{\mu}$ respectively. Also,

$$
L_{\Phi}=\left|D_{\lambda} \Phi\right|^{2} \equiv\left|\left(\partial_{\lambda}-\frac{i g}{2} \tau^{a} W_{\lambda}^{a}-\frac{i g^{\prime}}{2} Y_{\lambda}\right) \Phi\right|^{2}
$$




$$
V(\Phi)=\lambda\left(\Phi^{\dagger} \Phi-\eta^{2} / 2\right)^{2}
$$

where,

$$
\Phi=\left(\begin{array}{c}
\phi^{+} \\
\phi
\end{array}\right)
$$

is a complex doublet.

In addition, the fermionic sector Lagrangian is:

$$
L_{f}=L_{l}+L_{q}
$$

where, the lepton and quark sector Lagrangians for a single family are:

$$
\begin{gathered}
L_{l}=-i \bar{\Psi} \gamma^{\mu} D_{\mu} \Psi-i \bar{e}_{R} \gamma^{\mu} D_{\mu} e_{R}+h\left(\bar{e}_{R} \Phi^{\dagger} \Psi+\bar{\Psi} \Phi e_{R}\right) \\
L_{q}=-i(\bar{u}, \bar{d})_{L} \gamma^{\mu} D_{\mu}\left(\begin{array}{c}
u \\
d
\end{array}\right)_{L}-i \bar{u}_{R} \gamma^{\mu} D_{\mu} u_{R}-i \bar{d}_{R} \gamma^{\mu} D_{\mu} d_{R} \\
-G_{d}\left[(\bar{u}, \bar{d})_{L}\left(\begin{array}{c}
\phi^{+} \\
\phi
\end{array}\right) d_{R}+\bar{d}_{R}\left(\phi^{-}, \phi^{*}\right)\left(\begin{array}{c}
u \\
d
\end{array}\right)_{L}\right] \\
-G_{u}\left[(\bar{u}, \bar{d})_{L}\left(\begin{array}{c}
-\phi^{*} \\
\phi^{-}
\end{array}\right) u_{R}+\bar{u}_{R}\left(-\phi, \phi^{+}\right)\left(\begin{array}{c}
u \\
d
\end{array}\right)_{L}\right]
\end{gathered}
$$

with $\phi^{-}=\left(\phi^{+}\right)^{*}$. The indices $L$ and $R$ refer to left- and right-handed components.

In our analysis we will only be dealing with one fermion family at a time and hence we shall not be considering the effects of family mixing such as occurs due to the KM matrix. For our purpose, this simplification is justified because the string configuration has vanishing charged currents. It is known that in the neutral current sector the gauge and mass matrices can be diagonalized simultaneously [11] and hence there are no complications involving family mixing't.

The covariant derivatives occuring in the electroweak Lagrangian are:

$$
D_{\mu} \Psi=D_{\mu}\left(\begin{array}{l}
\nu \\
e
\end{array}\right)_{L}=\left(\partial_{\mu}-\frac{i g}{2} \tau^{a} W_{\mu}^{a}+\frac{i g^{\prime}}{2} Y_{\mu}\right)\left(\begin{array}{l}
\nu \\
e
\end{array}\right)_{L}
$$

\footnotetext{
${ }^{2}$ The decay of $Z$-strings necessarily involves the charged current sector and we expect family mixing effects (such as $\mathrm{CP}$ violation) to be present in such processes.
} 


$$
\begin{gathered}
D_{\mu} e_{R}=\left(\partial_{\mu}+i g^{\prime} Y_{\mu}\right) e_{R} \\
D_{\mu}\left(\begin{array}{l}
u \\
d
\end{array}\right)_{L}=\left(\partial_{\mu}-\frac{i g}{2} \tau^{a} W_{\mu}^{a}-\frac{i g^{\prime}}{6} Y_{\mu}\right)\left(\begin{array}{l}
u \\
d
\end{array}\right)_{L} \\
D_{\mu} u_{R}=\left(\partial_{\mu}-\frac{i 2 g^{\prime}}{3} Y_{\mu}\right) u_{R} \\
D_{\mu} d_{R}=\left(\partial_{\mu}+\frac{i g^{\prime}}{3} Y_{\mu}\right) d_{R}
\end{gathered}
$$

The conventions used here are those in Ref. [11].

We also need to define

$$
Z_{\mu} \equiv \cos \theta_{W} n^{a} W_{\mu}{ }^{a}-\sin \theta_{W} Y_{\mu}, \quad A_{\mu} \equiv \sin \theta_{W} n^{a} W_{\mu}{ }^{a}+\cos \theta_{W} Y_{\mu}
$$

where, $n^{a}$ is the unit vector

$$
n^{a}=-\frac{\Phi^{\dagger} \tau^{a} \Phi}{\Phi^{\dagger} \Phi}
$$

and,

$$
\tan \theta_{W} \equiv \frac{g^{\prime}}{g}, \quad \alpha \equiv\left(g^{2}+g^{\prime 2}\right)^{1 / 2} .
$$

For our purposes, only the $Z$ gauge field is non-zero and all the other gauge fields can be set to zero. This effectively reduces the bosonic part of the Lagrangian to an Abelian-Higgs model.

There are two distinct string solutions to the bosonic sector of the model - the $W$ and $Z$ strings [12, 13]. Here we shall only consider the unit winding $Z$ string for which the solution is:

$$
\Phi=\frac{\eta}{\sqrt{2}} f(r) e^{i \theta}\left(\begin{array}{l}
0 \\
1
\end{array}\right), \quad Z_{\theta}=-\frac{2}{\alpha} \frac{v(r)}{r}
$$

with all other fields set to zero. The solution in eqn. (21) is given in cylindrical coordinates $(r, \theta, z)$ and the profile functions $f$ and $v$ satisfy:

$$
\begin{gathered}
f^{\prime \prime}+\frac{f^{\prime}}{r}-\frac{f}{r^{2}}(1-v)^{2}+\lambda \eta^{2}\left(1-f^{2}\right) f=0 \\
v^{\prime \prime}-\frac{v^{\prime}}{r}+\frac{\alpha^{2} \eta^{2}}{4} f^{2}(1-v)=0
\end{gathered}
$$




$$
f(0)=v(0)=0, \quad f(\infty)=v(\infty)=1
$$

Note that in writing eqn. (22) we are only considering the bosonic sector and have set the fermionic fields to zero.

The total $Z$ magnetic flux in a string can be evaluated directly from eqns. (21) and (24):

$$
F_{Z}=\frac{4 \pi}{\alpha} .
$$

This means that any particle whose $Z$-charge is not an integer multiple of $\alpha / 2$ will have an Aharanov-Bohm interaction with the $Z$-string.

In Ref. [8], the baryon number on linked strings (Fig. 1) was calculated by integrating out the anomalous baryonic current conservation equation:

$$
\partial_{\mu} j_{B}^{\mu}=\frac{N_{F}}{32 \pi^{2}}\left[-g^{2} W_{\mu \nu}^{a} \tilde{W}^{a \mu \nu}+g^{\prime 2} Y_{\mu \nu} \tilde{Y}^{\mu \nu}\right]
$$

where, $N_{F}$ is the number of families and $\tilde{W}^{a \mu \nu} \equiv(1 / 2) \epsilon^{\mu \nu \lambda \sigma} W_{\lambda \sigma}^{a}$. The result iss:

$$
Q_{B}=2 N_{F} \cos \left(2 \theta_{w}\right)
$$

up to the addition of an integer. This calculation is indirect because it makes no explicit reference to the fermions. In Sec. 5 we shall recover (27) directly by studying the fermionic zero modes on the string.

The Dirac equations for a single family of leptons and quarks have been solved in the background of a straight $Z$ string by Earnshaw and Perkins [7]. The representation for the $\gamma$ matrices they use is:

$$
\begin{gathered}
\gamma^{r}=\left(\begin{array}{cccc}
0 & e^{-i \theta} & 0 & 0 \\
-e^{i \theta} & 0 & 0 & 0 \\
0 & 0 & 0 & -e^{-i \theta} \\
0 & 0 & e^{i \theta} & 0
\end{array}\right), \quad \gamma^{\theta}=\left(\begin{array}{cccc}
0 & -i e^{-i \theta} & 0 & 0 \\
-i e^{i \theta} & 0 & 0 & 0 \\
0 & 0 & 0 & i e^{-i \theta} \\
0 & 0 & i e^{i \theta} & 0
\end{array}\right), \\
\gamma^{0}=\left(\begin{array}{cc}
\tau^{3} & 0 \\
0 & -\tau^{3}
\end{array}\right), \quad \gamma^{z}=\left(\begin{array}{cc}
0 & \mathbf{1} \\
-\mathbf{1} & 0
\end{array}\right), \quad \gamma^{5}=\left(\begin{array}{ll}
0 & \mathbf{1} \\
\mathbf{1} & 0
\end{array}\right) .
\end{gathered}
$$

\footnotetext{
${ }^{3}$ We have included the factor of 2 error in Ref. [8] that is reported in the errata.
} 
For the electron they find the zero mode solution'f:

$$
e_{L}=\left(\begin{array}{c}
1 \\
0 \\
-1 \\
0
\end{array}\right) \psi_{1}(r), \quad e_{R}=\left(\begin{array}{l}
0 \\
1 \\
0 \\
1
\end{array}\right) i \psi_{4}(r)
$$

where,

$$
\begin{gathered}
\psi_{1}^{\prime}+\frac{q v}{r} \psi_{1}=-h \frac{\eta}{\sqrt{2}} f \psi_{4} \\
\psi_{4}^{\prime}-\frac{(q-1) v}{r} \psi_{4}=-h \frac{\eta}{\sqrt{2}} f \psi_{1} .
\end{gathered}
$$

$q$ denotes the $Z$ charge of the various left-handed fermions and for the electron we have $q=\cos \left(2 \theta_{W}\right)$. The boundary conditions are that $\psi_{1}$ and $\psi_{4}$ should vanish asymptotically. This means that there is only one arbitrary constant of integration in the solution to eqns. (31) and (32). This may be taken to be a normalization of $\psi_{1}$ and $\psi_{4}$.

Earnshaw and Perkins have also solved the Dirac equations for the quark zero modes. For the $d$ quark, the solution is the same as in eqns. (30), (31) and (32) except that $q=1-(2 / 3) \sin ^{2} \theta_{W}$. For the $u$ quark the solution is:

$$
u_{L}=\left(\begin{array}{c}
0 \\
1 \\
0 \\
-1
\end{array}\right) \psi_{2}(r), \quad u_{R}=\left(\begin{array}{l}
1 \\
0 \\
1 \\
0
\end{array}\right) i \psi_{3}(r)
$$

where,

$$
\begin{gathered}
\psi_{2}^{\prime}-\frac{q v}{r} \psi_{2}=-G_{u} \frac{\eta}{\sqrt{2}} f \psi_{3} \\
\psi_{3}^{\prime}+\frac{(q+1) v}{r} \psi_{3}=-G_{u} \frac{\eta}{\sqrt{2}} f \psi_{2}
\end{gathered}
$$

where, $q=-1+(4 / 3) \sin ^{2} \theta_{W}$. Note that (31), (32) are related to (34), (35) by $q \rightarrow-q$.

The neutrino zero modes can be found explicitly in terms of the string profile functions since the neutrino is massless and the right-hand sides of

\footnotetext{
${ }^{4}$ We have corrected an error of a minus sign in eqn. (19) of Ref. [7].
} 
the neutrino Dirac equations (corresponding to eqns. (34) and (35)) vanish. This is done in the next section.

We are mainly interested in the situation where two loops of string are linked (Fig. 1). To analyse this situation, we consider two circular loops of string of winding number $n$ that are linked but the strings themselves are far from each other. The loops are taken to be very much larger than the thickness of the strings and we shall always be working to lowest order in the ratio $w / a$ where $w$ is the string thickness and $a$ is the radius of the circular loops. In this approximation, we can ignore the curvature of the loop and consider the strings as being straight on scales much larger than $w$ but much smaller than $a$. Then the effect of one loop on the other is to produce a winding Higgs field and a winding gauge field with zero field strength at the location of the second loop. We can write down the field configuration of the linked loops in terms of the Higgs and gauge fields for two large loops in this approximation. If $\left(\phi^{(1)}, Z_{\mu}^{(1)}\right)$ and $\left(\phi^{(2)}, Z_{\mu}^{(2)}\right)$ are the fields for two isolated loops then the fields for the full configuration are given by the product ansatz:

$$
\phi=\frac{\sqrt{2}}{\eta} \phi^{(1)} \phi^{(2)}, \quad Z_{\mu}=Z_{\mu}^{(1)}+Z_{\mu}^{(2)} .
$$

If we set up a local coordinate frame on loop 1, with the $z$ axis along the string, the fields in the vicinity of the string can be found by using (21):

$$
\phi=\phi^{(1)} e^{i n z / a}, \quad Z_{\mu}=Z_{\mu}^{(1)}-\frac{2 n}{\alpha a} \hat{e}_{z} .
$$

\section{Fermionic Zero Mode Solutions}

In this section we give explicit solutions to the Dirac equations in (31) and (32) in two special cases. The first case is when the fermion is massless and the second case is when the fermion mass is equal to the scalar mass which is also equal to the vector mass. The first of these is relevant to neutrino zero modes. The second is not relevant to the electroweak case but may be of interest in other situations. 
We start out by writing eqns. (31) and (32) in more compact form by defining:

$$
F_{1}=\exp \left[+q \int d r \frac{v}{r}\right] \psi_{1}, \quad F_{4}=\exp \left[-(q-1) \int d r \frac{v}{r}\right] \psi_{4}
$$

Then,

$$
F_{1}^{\prime}=-\frac{h \eta f}{\sqrt{2}} \exp \left[+(2 q-1) \int d r \frac{v}{r}\right] F_{4}, \quad F_{4}^{\prime}=-\frac{h \eta f}{\sqrt{2}} \exp \left[-(2 q-1) \int d r \frac{v}{r}\right] F_{1} .
$$

For arbitrary parameters, the integral occuring in (39) is not known. However, in the Bogomolnyi limit - when the Higgs mass $\left(m_{H}=\sqrt{2 \lambda} \eta\right)$ and the $Z$ boson mass $\left(m_{Z}=\alpha \eta / 2\right)$ are equal - we can perform the integration. This is because, in this case, the equations for the string are equivalent to the following first order differential equations [10]:

$$
\begin{gathered}
f^{\prime}=\frac{f}{r}(1-v) \\
v^{\prime}=\frac{m_{Z}^{2}}{2} r\left(1-f^{2}\right) .
\end{gathered}
$$

Now eqn. (40) yields:

$$
\int d r \frac{v}{r}=\ln \left(\frac{m_{Z} r}{f}\right)
$$

where we have included a factor of $m_{Z}$ to make the argument of the logarithm dimensionless.

Therefore, when the fermion is massless $(h=0)$, the solutions are simply

$$
\psi_{1}=c_{1} m_{Z}^{3 / 2}\left(\frac{m_{Z} r}{f}\right)^{-q}, \quad \psi_{4}=c_{4} m_{Z}^{3 / 2}\left(\frac{m_{Z} r}{f}\right)^{q-1}
$$

where, $c_{1}$ and $c_{4}$ are independent constants that can be chosen to normalize the left- and right-handed fermion states and the spinors are given in (30). The boundary condition that the left-handed fermion wavefunction should vanish at infinity is only satisfied if $q>0$. Hence (43) can only give a valid solution for $q>0$ for the left-handed fermion. If we also require normalizability, we need $q>1$. (Note that there is no singularity at $r=0$ because $f \propto r$ when $r \sim 0$.) If we have a left-handed fermion with $q \leq-1$, the correct 
equations to use are the equations corresponding to the up quark equations given in (34) and (35) and these are solved by letting $q \rightarrow-q$ in (43). In this case, the spinors are given in (33).

For the neutrino, the right-handed component is absent and $q=-1$. This means that the neutrino has the same spinor structure as the left-handed up quark and the solution is that in (43) with $q$ replaced by +1 . Therefore the wave function falls off as $1 / r$ and the state is strictly not normalizable - the normalization integral diverges logarithmically. However, depending on the physical situation, one could be justified in imposing a cut-off. We shall be considering closed loops of string and the cutoff in our case is given by the radius of the loop.

Next, we consider the "super-Bogomolnyi" limit when the mass of the fermion $\left(m_{f}=h \eta / \sqrt{2}\right)$ is also equal to the Higgs and $Z$ boson masses. In this case, when the charge on the left-handed fermion vanishes $(q=0)$, by staring at the eqns. (39) with (42), (40) and (41) we guessed the solution to be:

$$
\begin{gathered}
\psi_{1}=N m_{Z}^{3 / 2}\left(1-f^{2}\right) \\
\psi_{4}=2 N m_{Z}^{1 / 2} \frac{f}{r}(1-v)
\end{gathered}
$$

where $N$ is a dimensionless normalization factor.

The solution for the up quark equations can be written down by using the transformation $q \rightarrow-q$ in the above solutions.

The solution in (44) and (45) can probably also be derived using supersymmetry arguments such as described by di Vecchia and Ferrara [15] but we are unaware of such a derivation in the literature.

The left-handed fermion wave-functions found above can be multiplied by a phase factor $\exp \left[i\left(E_{p} t-p z\right)\right.$ and the resulting wave-function will still solve the Dirac equations provided

$$
E_{p}=\epsilon_{i} p
$$

where, $i$ labels the fermions, and,

$$
\epsilon_{\nu}=+1=\epsilon_{u}, \quad \epsilon_{e}=-1=\epsilon_{d}
$$

In other words, $\nu_{L}$ and $u$ travel parallel to the string flux while $e$ and $d$ travel anti-parallel to the string flux. 


\section{Bosonic Condensates on Linked Strings}

Before proceeding to the fermionic case, let us consider the simpler case of bosonic superconductivity. If $\mathrm{A}$ and $\mathrm{B}$ are two loops of string linked to each other, then we shall see that there is a current induced in loop A provided that the bosonic condensate within its core has fractional charge with respect to the "magnetic" flux trapped in B.

The model we consider is the original Witten model [3] modified in order to allow for the Aharonov-Bohm-type interaction. We have two complex scalar fields $\Sigma$ and $\Phi$ and two $\mathrm{U}(1)$ gauge fields $A_{\mu}$ and $B_{\mu}$. The Lagrangian is

$$
\mathcal{L}=\left|D_{\mu} \Phi\right|^{2}+\left|D_{\mu} \Sigma\right|^{2}-\frac{1}{4} A_{\mu \nu} A^{\mu \nu}-\frac{1}{4} B_{\mu \nu} B^{\mu \nu}-\mathcal{V}(|\Phi|,|\Sigma|) .
$$

Here, $D_{\mu} \Phi=\left(\partial_{\mu}+i(q / 2) B_{\mu}\right) \Phi, D_{\mu} \Sigma=\left(\partial_{\mu}+i(e / 2) A_{\mu}+i\left(q^{\prime} / 2\right) B_{\mu}\right) \Sigma, A_{\mu \nu}=$ $\partial_{\mu} A_{\nu}-\partial_{\nu} A_{\mu}$ and $B_{\mu \nu}=\partial_{\mu} B_{\nu}-\partial_{\nu} B_{\mu}$. This Lagrangian is invariant under $U(1) \times U(1)$ gauge transformations:

$$
\Sigma \rightarrow \Sigma e^{-i q^{\prime} \Lambda}, \quad \Phi \rightarrow \Phi e^{-i q \Lambda}, \quad B_{\mu} \rightarrow B_{\mu}+2 \Lambda,_{\mu},
$$

and:

$$
\Sigma \rightarrow \Sigma e^{-i e \tilde{\Lambda}}, \quad A_{\mu} \rightarrow A_{\mu}+2 \tilde{\Lambda}_{, \mu},
$$

where $\Lambda$ and $\tilde{\Lambda}$ are arbitary functions. The potential

$$
\mathcal{V}=\lambda_{\phi}\left(|\Phi|^{2}-\eta^{2}\right)^{2}+f\left(|\Phi|^{2}-\eta^{2}\right)|\Sigma|^{2}+\lambda_{\sigma}|\Sigma|^{4}+m^{2}|\Sigma|^{2}
$$

is chosen so that the field $\Phi$ acquires a vacuum expectation value. Since the vacuum manifold is non-trivial, $\Phi$ will admit string-like solutions. The field $A_{\mu}$ is then identified with electromagnetism, which is unbroken outside the string, and $\Sigma$ plays the role of a charge carrier.

Straight string solutions can be studied in cylindrical coordinates using the ansatz

$$
\Phi=\phi(r) e^{i n \theta}, \quad B=B_{\theta}(r) d \theta, \quad \Sigma=\sigma(r), \quad A=A_{\theta}(r) d \theta
$$


where $n$ is an integer (the winding number). The field equations then take the form

$$
\begin{gathered}
\phi^{\prime \prime}+\frac{\phi^{\prime}}{r}-\frac{1}{r^{2}}\left(n+\frac{q}{2} B_{\theta}\right)^{2} \phi=\frac{1}{2} \mathcal{V},{ }_{\phi}, \\
\sigma^{\prime \prime}+\frac{\sigma^{\prime}}{r}-\frac{1}{r^{2}}\left(\frac{e}{2} A_{\theta}+\frac{q^{\prime}}{2} B_{\theta}\right)^{2} \sigma=\frac{1}{2} \mathcal{V},{ }_{\sigma}, \\
B_{\theta}^{\prime \prime}-\frac{B_{\theta}^{\prime}}{r}=q \phi^{2}\left(n+\frac{q}{2} B_{\theta}\right)+q^{\prime} \sigma^{2}\left(\frac{e}{2} A_{\theta}+\frac{q^{\prime}}{2} B_{\theta}\right), \\
A_{\theta}^{\prime \prime}-\frac{A_{\theta}^{\prime}}{r}=e \sigma^{2}\left(\frac{e}{2} A_{\theta}+\frac{q^{\prime}}{2} B_{\theta}\right) .
\end{gathered}
$$

With $\sigma=0$, these equations admit the usual Nielsen-Olesen vortex solutions [16]. The function $\phi(r)$ vanishes for $r \rightarrow 0$ and it approaches its vacuum expectation value $\eta$ exponentially fast for $r>\delta$, where $\delta \sim \lambda_{\phi}^{-1 / 2} \eta^{-1}$ is the thickness of the string core. The gauge field outside the core is given by

$$
B_{\theta}=-2 n / q,
$$

so that $D_{\mu} \Phi=0$ for $r>>\delta$. Therefore the flux of the $B_{\mu}$ field is quantized,

$$
F_{B}=4 \pi n / q
$$

and this will be important later on.

Although $\sigma=0$ is always a solution, Witten [3] showed that there is a range of parameters for which this solution is unstable. For $f \eta^{2}>m^{2}>0$ the effective mass squared for $\sigma$ is negative in the region where $\phi=0$, so that a condensate $\sigma \neq 0$ typically forms in the core of the string. To introduce a current along the string we need to consider "low energy" excitations on top of the condensate, of the form

$$
\Sigma=\sigma(r) e^{i \psi(z, t)}
$$

In the Lorentz gauge, the equation of motion for $\Sigma$ splits into a real and an imaginary part

$$
\begin{aligned}
\Delta \sigma-V_{\mu} V^{\mu} \sigma & =\frac{1}{2} \mathcal{V},_{\sigma} \\
\psi_{, t t}-\psi_{, z z} & =0
\end{aligned}
$$


where $V_{\mu} \equiv \psi_{, \mu}+\frac{e}{2} A_{\mu}+\frac{q^{\prime}}{2} B_{\mu}$ and $\Delta$ is the transverse Laplacian. The electromagnetic current on the string is given by

$$
J_{\mu}=\frac{\partial \mathcal{L}}{\partial A^{\mu}}=e \sigma^{2}\left(\psi_{, \mu}+\frac{e}{2} A_{\mu}+\frac{q^{\prime}}{2} B_{\mu}\right)
$$

Note that the phase $\psi$ obeys a massless wave equation in the longitudinal space, whose general solution is $\psi=f(t+z)+g(t-z)$, with $f$ and $g$ arbitrary functions. However, if we are interested in exact solutions within a simple ansatz (see below), we have to restrict attention to one of the following special cases: $\psi \propto t$, corresponding to a constant charge per unit length, $\psi \propto z$, corresponding to a constant space-like current, or one of the forms $\psi=f(t+z), \psi=g(t-z)$, corresponding to light-like currents of arbitrary profile propagating along the string [17]. More general forms for $\psi$ will radiate and settle down to one of these special cases. For our problem, the relevant configuration will be the one with a space-like current, $\psi \propto z$.

The reason why linked loops must carry non-vanishing currents can be seen from Eq.(61). Neglecting, for the time being, the contribution of $A_{\mu}$, the circulation of $J_{\mu}$ along one of the loops is proportional to

$$
\oint \psi,{ }_{\mu} d x^{\mu}+\frac{q^{\prime}}{2} \oint B_{\mu} d x^{\mu}=\Delta \psi+\frac{2 \pi q^{\prime}}{q}
$$

where we have used that the circulation of $B_{\mu}$ along one loop is equal to the flux (58) trapped in the core of the other loop. Since $\Sigma$ has to be single valued, $\Delta \psi$ must be a multiple of $2 \pi$. Therefore $J_{\mu}$ cannot vanish unless $q^{\prime} / q$ is an integer.

Let us now make a more quantitative estimate of the actual current induced on the loops. As mentioned in section 2, to lowest order in $w / a$, we can mimick the linkage by taking just one straight string along the z-axis with appropriate periodic boundary conditions. To this end, we generalize the ansatz (52), allowing for a twist in the $\Phi$ field (see (37)):

$$
\Phi=\phi(r) e^{i n \theta} e^{i n z / a}
$$

Identifying $z=-\pi a$ with $z=\pi a$, this will represent a loop of radius $a$ and winding number $n$ which is being threaded by another string of winding number $n$, as in Fig. 1. 
In the presence of a $z$ dependent phase in (59), it is necessary to introduce longitudinal components $A_{z}(r)$ and $B_{z}(r)$ for the gauge fields. Then, in addition to eqns. (55), (56) and (50) above, we have

$$
\begin{gathered}
B_{z}^{\prime \prime}+\frac{1}{r} B_{z}^{\prime}=q \phi^{2}\left(\frac{n}{a}+\frac{q}{2} B_{z}\right)+\frac{q^{\prime}}{2} \sigma^{2} V_{z}, \\
A_{z}^{\prime \prime}+\frac{1}{r} A_{z}^{\prime}=e \sigma^{2} V_{z} \\
\phi^{\prime \prime}+\frac{\phi^{\prime}}{r}-\left[\left(\frac{n}{a}+\frac{q}{2} B_{z}\right)^{2}+\frac{1}{r^{2}}\left(n+\frac{q}{2} B_{\theta}\right)^{2}\right] \phi=\frac{1}{2} \mathcal{V},_{\phi} .
\end{gathered}
$$

As with the $\theta$ component, the condition that $D_{z} \Phi$ vanishes outside the core of the string yields the asymptotic value $B_{z}=-2 n /(a q)$. This represents the pure gauge winding around the 'other' string.

The current flowing along the string will be given, from (61), by

$$
J=K e\left(\psi,{ }_{z}+\frac{e}{2} \bar{A}_{z}+\frac{q^{\prime}}{2} \bar{B}_{z}\right) .
$$

Here $K \equiv \int d^{2} x \sigma^{2}$, where the integral is over the transverse section of the string, $\psi=l z / a$, where $l$ is an integer, and $\bar{A}_{z}, \bar{B}_{z}$ are the gauge fields averaged over the cross section of the core with weight given by $\sigma^{2}$. To lowest order in the gauge couplings, $\bar{B}_{z}$ is given by its asymptotic value $-2 n /(a q)$, as indicated above. Following Witten [3], in order to estimate $\bar{A}_{z}$ we consider the formal solution of Eq. (63). For an idealized infinitely thin current we have

$$
A_{z}(\mathbf{x})=-\frac{1}{4 \pi} \int d z \frac{1}{|\mathbf{x}-\mathbf{x}(z)|} J(z) .
$$

The logarithmic divergence in the right hand side of (65) can be regularized by considering a current of finite thickness $m_{\sigma}^{-1}$. Then, along the string

$$
\bar{A}_{z} \approx-\frac{1}{2 \pi} \ln \left(m_{\sigma} a\right) J
$$

where $J$ is given by (64). Eliminating $\bar{A}_{z}$ from both equations we have (to lowest order in gauge couplings)

$$
J \approx \frac{K e}{a\left[1+K\left(e^{2} / 4 \pi\right) \ln \left(m_{\sigma} a\right)\right]}\left(l-n \frac{q^{\prime}}{q}\right),
$$


And, neglecting unity in front of the logarithmic term,

$$
\bar{A}_{z} \approx \frac{4 \pi}{a e}\left(l-n \frac{q^{\prime}}{q}\right) .
$$

Therefore, if $n q^{\prime} / q$ is not an integer, we reach the conclusion that there has to be at least a current of magnitude $J \sim\left(\text { ae } \ln \left[m_{\sigma} a\right]\right)^{-1}$ flowing along the string.

A new interesting phenomenon can be seen if we go beyond lowest order in $e$. Note that for $q^{\prime} \neq 0$ there has to be a magnetic field $A_{r \theta} \neq 0$ trapped in the core of the string, since Eq. (56) has a source term of the form $e \sigma^{2} q^{\prime} B_{\theta}$. This source term corresponds to a solenoid-like current that wraps around the outer layers of the string core. Since the thickness of the condensate is of order $\left(m_{\sigma}\right)^{-1} \equiv\left(\eta^{2} f-m^{2}\right)^{-1 / 2} \gtrsim \delta$, and the asymptotic value of $B_{\theta}$ is given by (57), the magnetic flux of $A_{\mu}$ trapped within the "solenoid" will be of order

$$
F_{A} \sim e n \frac{q^{\prime}}{q} \frac{\sigma_{0}^{2}}{m_{\sigma}^{2}},
$$

where $\sigma_{0} \sim \lambda^{-1 / 2} m_{\sigma}$ is the magnitude of the condensate. Depending on the parameters, this flux can be quite large and have interesting consequences.

In particular, when two loops are linked it is not possible to have the circulation of $A_{\mu}$ vanish along the loops, and a current will arise. This effect is of order $e^{2} / \lambda_{\sigma}$ compared to the lowest order contribution (66), but can give the dominant effect if $n q^{\prime} / q$ is an integer (in which case we can set $l=n q^{\prime} / q$ ), or if the self coupling $\lambda_{\sigma}$ is sufficiently small.

\section{$5 \quad$ Fermionic Zero Modes on Linked Strings}

The Dirac equations that we need to solve for the electron and $d$ quark are of the general form:

$$
i \gamma^{\mu} D_{\mu}^{(q)} \psi_{L}=h \phi \psi_{R}, \quad i \gamma^{\mu} D_{\mu}^{(q-1)} \psi_{R}=h \phi^{*} \psi_{L}
$$


where, $D_{\mu}^{(q)}=\partial_{\mu}+i q Z_{\mu}$ and the bosonic background is given in (37). For the $u$ quark, $\phi$ must be replaced by $\phi^{*}$ and $q-1$ by $q+1$ in the second equation. For the neutrino we should set $h=0$.

The Dirac equations (68) split up into equations in the transverse directions to the string and in the longitudinal direction. In what follows, we will only be considering the zero mode solutions to the transverse part of the Dirac equation as these are the low energy modes that live on the string and are the dominant new effect due to the string background. The other massive modes would also feel the string background but we expect the effects due to these modes to be smaller. A rigorous analysis, however, would have to take all the transverse modes, including the massive ones, into account.

To solve eqn. (68), we consider the ansatz

$$
\psi_{L}=e^{-i\left(E_{p} t-p z\right)} \psi_{L}^{(0)}(r), \quad \psi_{R}=e^{-i\left(E_{p} t-(p-n / a) z\right)} \psi_{R}^{(0)}(r)
$$

where, the superscript $(0)$ denotes that the function is the one found on the straight string as described in sections 2 and 3 and $n$ denotes the winding number of the two strings (assumed equal). The coordinates $(t, r, \theta, z)$ are cylindrical coordinates with the string locally along the $z$ axis and we are confining our attention to the vicinity of a point on one of the strings. The functions $\psi_{L}^{(0)}$ and $\psi_{R}^{(0)}$ should be normalized so that

$$
\int d^{3} x \psi^{\dagger} \psi=\int d^{3} x\left(\left|\psi_{L}^{(0)}\right|^{2}+\left|\psi_{R}^{(0)}\right|^{2}\right)=1
$$

This condition completely fixes the fermion wavefunction since there is only one arbitrary normalization factor in the solution. (For example, see eqns. (44) and (45).) But without an explicit solution, we cannot say what the normalization of the left-handed component is relative to the right-handed component.

To lowest order in $w / a$, eqn. (69) is a solution provided $E$ and $p$ satisfy the dispersion relation:

$$
E_{p}=\epsilon_{i}\left(p+q Z_{z}\right)
$$

where, the symbol $\epsilon_{i}$ is defined below eqn. (46), $Z_{z}$ is given by (37) and $a p$ has to be an integer for single-valuedness of the wavefunctions. So, in terms of $\omega \equiv a E$ and $k \equiv a p \in \mathcal{Z}$, we have,

$$
\omega_{k}=\epsilon_{i}(k-q Z) \text {. }
$$


where,

$$
Z \equiv \frac{2 n}{\alpha}
$$

The crucial property of this equation is that, if there is an Aharanov-Bohm interaction between the $Z$-string and the fermion, $\omega_{k}$ cannot be zero for any value of $k$ since $k$ is an integer.

The energy of the fermions is found by summing over the negative frequencies - that is, the Dirac sea - and so the energy $E$ is:

$$
E=\frac{1}{a} \sum_{\omega_{k}<0} \omega_{k}=\epsilon_{i} \frac{1}{a} \sum_{\omega_{k}<0}(k-q Z)=\epsilon_{i} \frac{1}{a} \sum_{k=k_{F}}^{-\epsilon_{i} \infty}(k-q Z)
$$

where, $k_{F}$ denotes the Fermi level - the value of $k$ for the highest filled state. Therefore we need to sum a series of the type:

$$
S=\sum_{k=k_{F}}^{\infty}(k-q Z)=\sum_{k=0}^{\infty}\left(k+k_{F}-q Z\right) .
$$

The sum is found using the zeta function regularization [18] :

$$
S=\zeta\left(-1, k_{F}-q Z\right)=-\frac{1}{12}-\frac{1}{2}\left(k_{F}-q Z\right)\left(k_{F}-q Z-1\right)
$$

With this result, the energy contribution from the fermions takes the form:

$$
E_{i}=-\frac{1}{24 a}+\frac{1}{2 a}\left[k_{F}^{(i)}-q_{i} Z+\frac{\epsilon_{i}}{2}\right]^{2} \equiv-\frac{1}{24 a}+\frac{1}{2 a} K_{i}^{2}
$$

where, the index $i$ is a label for the particular fermion in question. As the non-trivial term in $E_{i}$ comes in the form of a square, the ground state can be found by minimizing $E_{i}$ with respect to $k_{F}^{(i)}$ for each $i$. The result of this exercise is summarized in Table 1 where we give the charges, the Fermi levels $k_{F}^{(i)}$ (for $\gamma=\sin ^{2} \theta_{W}$ close to $1 / 4$ ) and the value of the minimum energy contribution for each fermion $\left(\nu_{L}, e, d, u\right)$ from one family. (The other families contribute identically.) If we now also include the fact that the quarks come in 3 colours and that we have 2 loops that are linked, the total energy is:

$$
E=2 N_{F}\left(E_{\nu}+E_{e}+3 E_{d}+3 E_{u}\right)=\frac{4 N_{F}}{3 a}\left[8 \gamma^{2}-6 \gamma+1\right]
$$

\footnotetext{
${ }^{5}$ The contribution $1 / 12 a$ is the Casimir energy since the strings are closed and is present whether the loops are linked or unlinked.
} 
Note that $E_{i}$ attains its maximum value of $1 / 12 a$ if $q_{i} Z$ is integral and its minimum value of $-1 / 24 a$ if $q_{i} Z$ is half integral. When the loops are unlinked, we have $q_{i} Z=0$ and so the ground state energy of unlinked loops has to be larger than or equal to the ground state energy of linked loops. An explicit calculation along the lines used for linked loops (eqn. (78)) for two unlinked loops yields:

$$
E(\text { unlinked })=\frac{4 N_{F}}{3 a}
$$

and so the difference of the unlinked and linked ground state energies is:

$$
\Delta E=\frac{8 N_{F}}{3 a} \gamma[4 \gamma-3] .
$$

Inserting physical values $\gamma=0.23$ and $N_{F}=3$, we get,

$$
\Delta E=-3.8 / a .
$$

Note that in calculating the energy (78), (79) we have included the contribution from the neutrinos by using the formula (77). As the neutrino is massless and the zero mode state is only normalizable on imposing a cut-off, it is an open question if it is legitimate to ignore the contribution of the other neutrino modes besides the zero mode. This is a subtle issue which has been discussed in the context of $2+1$ dimensions in the existing literature [19]. In the present context, we are considering closed string loops in $3+1$ dimensions and the issue deserves further investigation. Here we will only remark that the question applies regardless of whether the loops are linked or not and so the issue has no bearing on the difference in energies between linked and unlinked loops given in (81).

The energy momentum for fermions can be found by varying the action with respect to the metric and is:

$$
T_{\mu \nu}=\frac{i}{2}\left[\bar{\psi} \gamma_{(\mu} D_{\nu)} \psi-\left(D_{(\mu} \psi\right)^{\dagger} \gamma^{0} \gamma_{\nu)} \psi\right] .
$$

This leads to the result that the magnitude of the fermion momentum along the string is equal to the energy of the fermion - a result which could also be deduced by noting that the fermions are massless on the string. Now to find the total momentum, we should recall that the electron and $d$ quark travel in one direction while the neutrino and $u$ quark travel in the opposite direction. 
Using this fact, we find that the magnitude of the angular momentum due to the fermionic ground state on one of the strings is:

$$
\Omega=a N_{F}\left(E_{\nu}-E_{e}-3 E_{d}+3 E_{u}\right)=0
$$

when the linkage is one.

In non-trivial but symmetric backgrounds, it is possible to define a generalized angular momentum operator for the fluctuations on top of the background. If we confine our attention to the case where we have a loop in the $x y$-plane and $n$ straight infinite strings along the $z$-axis threading the circular loop, this system has rotational symmetry about the $z$-axis. The operator that annihilates the field configuration is the generalized angular momentum operator and is 20

$$
M_{z}=L_{z}+S_{z}+n I_{z}
$$

where,

$$
L_{z}=-i 1 \frac{\partial}{\partial \theta}
$$

$S_{z}$ is the spin operator, and, the isospin operator is given in terms of the $U(1)$ (hypercharge) and $S U(2)$ charges - $q_{1}$ and $q_{2}$ respectively - of the field in question:

$$
I_{z}=\frac{1}{2}\left[\left(\frac{2 q_{2}}{g}\right) \tau^{3}-\left(\frac{2 q_{1}}{g^{\prime}}\right) \mathbf{1}\right] .
$$

The isopin operator acts via a commutator bracket on the gauge fields and by ordinary matrix multiplication on the Higgs field and fermion doublets.

We are interested in the angular momentum of the fermions on the circular loop which lies entirely in the $x y$-plane. The fermions in the zero modes therefore have $S_{z}=0$. The action of $L_{z}$ is found by acting on the fermion wave-functions such as in (69) (remembering to let $n \rightarrow-n$ for the neutrino and up quark). The action of $I_{z}$ is found by using the charges of the fermions given in (13)-(17). We then find:

$$
\begin{gathered}
M_{z}\left(\begin{array}{c}
\nu_{L} \\
e_{L}
\end{array}\right)=\left(\begin{array}{c}
\left(k^{(\nu)}+n\right) \nu_{L} \\
k^{(e)} e_{L}
\end{array}\right), \quad M_{z}\left(\begin{array}{c}
u_{L} \\
d_{L}
\end{array}\right)=\left(\begin{array}{c}
\left(k^{(u)}+\frac{n}{3}\right) u_{L} \\
\left(k^{(d)}-\frac{2 n}{3}\right) d_{L}
\end{array}\right), \\
M_{z} e_{R}=k^{(e)} e_{R}, \quad M_{z} u_{R}=\left(k^{(u)}+\frac{n}{3}\right) u_{R}, \quad M_{z} d_{R}=\left(k^{(d)}-\frac{2 n}{3}\right) d_{R}
\end{gathered}
$$


where the $k^{(i)}$ are defined above eqn. (72). Now summing over states, as in the case of the energy, we find the total generalized angular momentum of the fermions on the circular loop:

$$
\mathcal{M}=\frac{1}{2}\left[k_{F}^{(\nu)}+n+\frac{1}{2}\right]^{2}-\frac{1}{2}\left[k_{F}^{(e)}-\frac{1}{2}\right]-\frac{3}{2}\left[k_{F}^{(d)}-\frac{2 n}{3}-\frac{1}{2}\right]^{2}+\frac{3}{2}\left[k_{F}^{(u)}+\frac{n}{3}+\frac{1}{2}\right]^{2} .
$$

Note that though the gauge fields do not enter explicitly in the generalized angular momentum, they do play a role in determining the angular momentum of the ground state through the values of the Fermi levels. If we now consider the case $n=1$, and use the values of the Fermi levels from Table 1, we find $\mathcal{M}=0$.

We now calculate the electromagnetic and baryonic charges and currents on the linked loops. For this we must sum over the charges of each filled state. Since the states up to the state $k_{F}^{(i)}$ are filled, we need to find a sum of the kind:

$$
S_{q}=\sum_{k=k_{F}}^{\infty} 1
$$

To regularize the divergence of the series, we write it as

$$
S_{q}=\lim _{\lambda \rightarrow 0} \sum_{k=k_{F}}^{\infty}(k-q Z)^{\lambda} .
$$

Note that we have chosen to use the gauge invariant combination $k-q Z$ rather than $k$ or some other gauge non-invariant expression [9]. Now we can use the zeta function regularization to get:

$$
S_{q}=\sum_{k=0}^{\infty}\left(k+k_{F}-q Z\right)^{0}=\zeta\left(0, k_{F}-q Z\right)=-\left[k_{F}-q Z-\frac{1}{2}\right] .
$$

With this result, we find the contribution to the charge due to fermion $i$ :

$$
Q_{i}=\epsilon_{i} q_{i}\left[k_{F}^{(i)}-q_{i} Z+\frac{\epsilon_{i}}{2}\right]=\epsilon_{i} q_{i} K_{i}
$$

The currents along the $z$ direction are given by $\bar{\psi} \gamma^{z} \psi$ where $\gamma^{z}$ is given in eqn. (29). This gives

$$
J_{i}=\epsilon_{i} Q_{i}
$$


Now we can evaluate the electric and baryon charges and currents for each of the fermions $e, d$ and $u$. The results are exhibited in Table 1 . Finally we can find the total charges and currents on the linked strings by adding up the contributions of the leptons and quarks and also taking into account that the quarks come in 3 colours, that we have 2 loops and $N_{F}$ families. The arithmetic gives the electric charge:

$$
Q_{A}=0 ;
$$

the electric current along one of the strings:

$$
J_{A}=2 e N_{F}\left(1-\frac{8}{3} \sin ^{2} \theta_{W}\right) ;
$$

the baryonic charge - in agreement with the results obtained by indirect methods [8]:

$$
B=2 N_{F} \cos \left(2 \theta_{W}\right) ;
$$

and the baryonic current along one of the strings:

$$
J_{B}=-\frac{2 N_{F}}{3} \sin ^{2} \theta_{W} .
$$

It is interesting to work out the changes that occur when the strings have linkage greater than 1 . We have considered the situation when a loop is threaded by $n$ other loops. In this case, the charges $q_{Z}$ in Table 1 have to be multiplied by $n$ and then this changes the Fermi levels. To analyze this situation, we begin by writing all the quantities of interest in terms of the $K_{i}$ for a single loop with $N_{F}$ set equal to 1 for convenience:

$$
\begin{gathered}
2 a E=K_{\nu}^{2}+K_{e}^{2}+3 K_{d}^{2}+3 K_{u}^{2} \\
2 \Omega=K_{\nu}^{2}-K_{e}^{2}-3 K_{d}^{2}+3 K_{u}^{2} \\
Q_{A} / e=K_{e}+K_{d}+2 K_{u} \\
B=-K_{d}+K_{u} \\
J_{A} / e=-K_{e}-K_{d}+2 K_{u} \\
J_{B}=K_{d}+K_{u}
\end{gathered}
$$


Table 1: Summary of $Z$-, electric and baryon charges, Fermi levels, energies, induced electric charges, baryon numbers and electric and baryonic currents for the leptons and quarks. The charges $q_{Z}$ are for the left-handed fermions. In evaluating the Fermi levels, we have taken unit winding strings and $\gamma \equiv$ $\sin ^{2} \theta_{W} \sim 0.23$.

\begin{tabular}{lcccc}
\hline & $\nu_{L}$ & $e$ & $d$ & $u$ \\
\hline $2 q_{Z} / \alpha$ & -1 & $1-2 \gamma$ & $1-\frac{2 \gamma}{3}$ & $-1+\frac{4 \gamma}{3}$ \\
$q_{A} / e$ & 0 & -1 & $-1 / 3$ & $2 / 3$ \\
$q_{B}$ & 0 & 0 & $1 / 3$ & $1 / 3$ \\
$k_{F}^{(i)}$ & -1 & +1 & +1 & -1 \\
$a E_{i}+\frac{1}{24}$ & $\frac{1}{8}$ & $\frac{1}{2}\left(2 \gamma-\frac{1}{2}\right)^{2}$ & $\frac{1}{2}\left(\frac{2 \gamma}{3}-\frac{1}{2}\right)^{2}$ & $\frac{1}{2}\left(\frac{4 \gamma}{3}-\frac{1}{2}\right)^{2}$ \\
$Q_{i}$ & 0 & $\left(2 \gamma-\frac{1}{2}\right)$ & $\frac{1}{3}\left(\frac{2 \gamma}{3}-\frac{1}{2}\right)$ & $-\frac{2}{3}\left(\frac{4 \gamma}{3}-\frac{1}{2}\right)$ \\
$B_{i}$ & 0 & 0 & $-\frac{1}{3}\left(\frac{2 \gamma}{3}-\frac{1}{2}\right)$ & $-\frac{1}{3}\left(\frac{4 \gamma}{3}-\frac{1}{2}\right)$ \\
$j_{i}^{A}$ & 0 & $-\left(2 \gamma-\frac{1}{2}\right)$ & $-\frac{1}{3}\left(\frac{2 \gamma}{3}-\frac{1}{2}\right)$ & $-\frac{2}{3}\left(\frac{4 \gamma}{3}-\frac{1}{2}\right)$ \\
$j_{i}^{B}$ & 0 & 0 & $\frac{1}{3}\left(\frac{2 \gamma}{3}-\frac{1}{2}\right)$ & $-\frac{1}{3}\left(\frac{4 \gamma}{3}-\frac{1}{2}\right)$ \\
\hline
\end{tabular}

where the $K_{i}$ are defined in general in eqn. (77). For the ground state, the Fermi levels are chosen to minimize the energy and then the $K_{i}$ can be written as:

$$
\begin{gathered}
K_{\nu}(x)=\left\{\left\{\frac{1}{2}\right\}\right\}, \\
K_{e}(x)=\left\{\left\{3 x-\frac{1}{2}\right\}\right\}, \\
K_{d}(x)=\left\{\left\{x-\frac{1}{2}\right\}\right\},
\end{gathered}
$$

and,

$$
K_{u}(x)=-\left\{\left\{2 x-\frac{1}{2}\right\}\right\}
$$

where $x=2 n \gamma / 3$ and $\{\{y\}\}$ is the smallest (in magnitude) fractional part of $y$. (For example, for $y=+0.2$ and +0.7 , these are 0.2 and -0.3 . For half-integral values of $y$, there is a sign ambiguity but this does not affect 
our results for the energy and angular momentum which are quadratic in $K_{i}$. The charges and currents can depend on the choice of sign but only at isolated points. For irrational values of $\sin ^{2} \theta_{W}, x$ can never take on these values. To find $\{\{y\}\}$ for negative $y$ we can use the property $\{\{-y\}\}=-\{\{y\}\}$.)

It is easy to check that

$$
K_{i}(x+k)=K_{i}(x), \quad k \in \mathcal{Z}
$$

and so we can restrict our attention to $x \in(0,1)$. In this interval, we can check that,

$$
\left\{\left\{m x-\frac{1}{2}\right\}\right\}=m x-\frac{1}{2}-i, \quad x \in\left(\frac{i}{m}, \frac{i+1}{m}\right)
$$

where, $m$ is an integer and $i=0,1, \ldots, m-1$. This general formula yields:

$$
K_{i}(1-x)=-K_{i}(x)
$$

and so we need only calculate the quantities of interest in the interval $(0,1 / 2)$. Outside this range, we can write down the values of the quantities by using the symmetry in (111).

In Table 2 we show the expressions for the energy, angular momentum, charges and currents for one loop threaded by $n$ strings in terms of $x=2 n \gamma / 3$. Note that the angular momentum is non-vanishing only when the electric charge is non-zero, and as defined by $\Omega$, does not come in half-integral units. This is of no concern because $\Omega$ does not include the angular momentum of the bosonic background and so can have any fractional value. On the other hand, the generalized angular momentum found in eqn. (89) is always halfintegral as can be seen, for example, by expanding out the right-hand side of (89). As shown in Table 2 , in the ground state, the baryon number of the single loop is given by $n N_{F} \cos 2 \theta_{W}$ up to an additive integer. This is also what we expect from integrating out the anomaly equation 8 .

The energy of the fermionic ground state shows a complicated dependence on $x$ as is demonstrated in Fig. 2. By changing the linking number, we can change $x$ in discrete steps and sample different points on the $E(x)$ curve. Since $E(x)$ does not have a monotonic dependence on $x$, the energy of strings that are linked $n$ times bears no simple relation to those linked $m$ times. In particular, the energy does not continue to decrease as we consider strings that have higher linkage. The lowest energy possible is when $x=1 / 4$ but, if 
Table 2: Expressions for the energy, angular momentum, charges and currents in terms of $x=2 n \gamma / 3$. We have omitted the multiplicative factor $N_{F}$ in all the expressions for convenience.

\begin{tabular}{lcccc}
\hline & $x \in(0,1 / 3)$ & $(1 / 3,1 / 2)$ & $(1 / 2,2 / 3)$ & $(2 / 3,1)$ \\
\hline$a E$ & $12 x^{2}-6 x+1$ & $12 x^{2}-9 x+2$ & $12 x^{2}-15 x+5$ & $12 x^{2}-18 x+7$ \\
$\Omega$ & 0 & $3 x-1$ & $-3 x+2$ & 0 \\
$Q_{A} / e$ & 0 & -1 & +1 & 0 \\
$B$ & $-3 x+1$ & $-3 x+1$ & $-3 x+2$ & $-3 x+2$ \\
$J_{A} / e$ & $-8 x+2$ & $-8 x+3$ & $-8 x+5$ & $-8 x+6$ \\
$J_{B}$ & $-x$ & $-x$ & $1-x$ & $1-x$ \\
\hline
\end{tabular}

$\gamma$ is irrational, there will not be any value of the linkage for which $x$ will be $1 / 4$. (Though we can choose values of $n$ for which $x$ is arbitrarily close to $1 / 4)$.

The curve in Fig. 2 has the interpretation of a potential for the quantity $x$ and tells us which values of $x$ are most favoured. In a physical situation, however, we should calculate the free energy which would be large for large linkage. This would remove the degeneracy between minima of different linkage and, for high enough string density, we would probably be left with two degenerate global minima - one occurring at some positive value of $x$ and another at the negative of this value.

\section{Conclusions}

In this paper we have studied boson and fermion zero modes on linked string configurations including the case when the boson or fermion has an Aharanov-Bohm interaction with the string. From this analysis we can calculate the various charges on the linked strings. We confirm that the baryon number on linked loops of electroweak string is that found in Ref. [8]: $2 N_{F} \cos \left(2 \theta_{W}\right)$. In addition, our direct calculations enable us to calculate the electric charge, electric current and electomagnetic current on the linked 
strings. The charge vanishes on singly linked unit winding string loops but the electric and baryonic currents are non-vanishing. For strings with higher linking numbers, the situation gets more complicated. While the energy of linked strings is always less than the energy of unlinked strings, the energy is not a monotonic function of the linking number and fluctuates as shown in Fig. 2. In the bosonic case, we also find that the ground state of the linked configuration is current carrying.

We have found a "super-Bogomolnyi" limit in which the Dirac equations for the fermions can be reduced by one order to an algebraic equation. This is the limit when the scalar, vector and fermion masses are all equal and the charge of the left-handed fermion vanishes. Probably this limit and the result are related to a manifestation of supersymmetry but we are not aware of a discussion of this point in the literature.

Finally, we propose that there may be a gravitational analogue of the system of linked strings. This is because a particle moving in a conical space - such as produced by a cosmic string - has a gravitational Aharanov-Bohm interaction with the line source that produces the conical space. Then if we consider two circular line sources each of fixed radius $a$, the total source length in the absence of linkage is simply $4 \pi a$. But if the sources are linked, the gravitational Aharanov-Bohm effect reduces the total length of the sources to $2(2 \pi-\delta) a$ where $\delta$ is the conical deficit angle produced by the sources. So the linked sources have less length for the same radius as compared to the unlinked sources. This is the gravitational analogue of the result that the ground state of linked strings is lower than the ground state of unlinked strings.

Even though there are traveling wave solutions ("zero modes") on gravitating strings [21], there does not seem to be any gravitational analogue of the currents that run along linked strings - at least, in the context of static, conical spaces in conventional general relativity. This is because there is nothing to distinguish one direction along the line source over the other. However, it may be that there are non-vanishing currents along the sources if the linked sources are spinning [22]. We feel that this system is worth exploring further. 


\section{Acknowledgements}

We are grateful to the steady stream of visitors at the Isaac Newton Institute for advice and suggestions. In particular, we would like to thank Tom Kibble, Patrick Peter, Rich Holman, Mark Hindmarsh, Rob Brandenberger, Roman Jackiw, Patricio Letelier, Hector de Vega and especially Nick Manton for crucial advice at critical times. JG is grateful to the SERC for support under Grant No. 15091-AOZ-L9 at DAMTP, University of Cambridge where most of this work was done. TV is grateful to the Rosenbaum Foundation for supporting his stay at the Isaac Newton Institute.

\section{References}

[1] R. Jackiw and C. Rebbi, Phys. Rev. D13, 3398 (1976).

[2] J. Goldstone and F. Wilczek, Phys. Rev. Lett. 47, 986 (1981).

[3] E. Witten, Nucl. Phys. B249, 557 (1985).

[4] Y. Nambu, Nucl. Phys. B130, 505 (1977).

[5] N. S. Manton, Phys. Rev. D28, 2019 (1983).

[6] T. Vachaspati, Phys. Rev. Lett. 68, 1977 (1992); 69, 216(E) (1992); Nucl. Phys. B397, 648 (1993).

[7] M. Earnshaw and W. Perkins, Phys. Lett. B328, 337 (1994).

[8] T. Vachaspati and G. B. Field, Phys. Rev. Lett. 73, 373 (1994).

[9] N. S. Manton, Ann. of Phys. 159, 220 (1985).

[10] E. B. Bogomolnyi, Sov. J. Nucl. Phys. 24, 449 (1976).

[11] T. P. Cheng and L. F. Li, "Gauge Theory of Elementary Particle Physics", Oxford University Press (1991).

[12] T. Vachaspati and M. Barriola, Phys. Rev. Lett. 69, 1867 (1992).

[13] M. Barriola, T. Vachaspati and M. Bucher, Phys. Rev. D50, 2819 (1994). 
[14] E. Weinberg, Phys. Rev. D24, 2669 (1981).

[15] P. di Vecchia and S. Ferrara, Nucl. Phys. B130, 93 (1977).

[16] H. B. Nielsen and P. Olesen, Nucl. Phys. B61, 45 (1973).

[17] J. Garriga and P. Peter, Class. Quant. Grav. 11, 1743 (1994).

[18] I.S. Gradshteyn and I.M. Ryzhik, Tables of integrals, series and products, Academic Press, New York (1980).

[19] H. de Vega, Phys. Rev. D18, 2932 (1978).

[20] M. James, L. Perivolaropoulos and T. Vachaspati, Nucl. Phys. B395, 534 (1993).

[21] D. Garfinkle and T. Vachaspati, Phys. Rev. D42, 1960 (1990).

[22] P.S. Letelier, Isaac Newton Institute preprint; to appear in Classical and Quantum Gravity (1994).

\section{Figure captions}

- Fig. 1 A pair of linked loops.

- Fig. 2 The fermionic ground-state energy $E$ (in units of $1 / a$ ) of a single loop of string threaded by $n$ other strings versus $x=(2 n / 3) \sin ^{2} \theta_{W}$. 


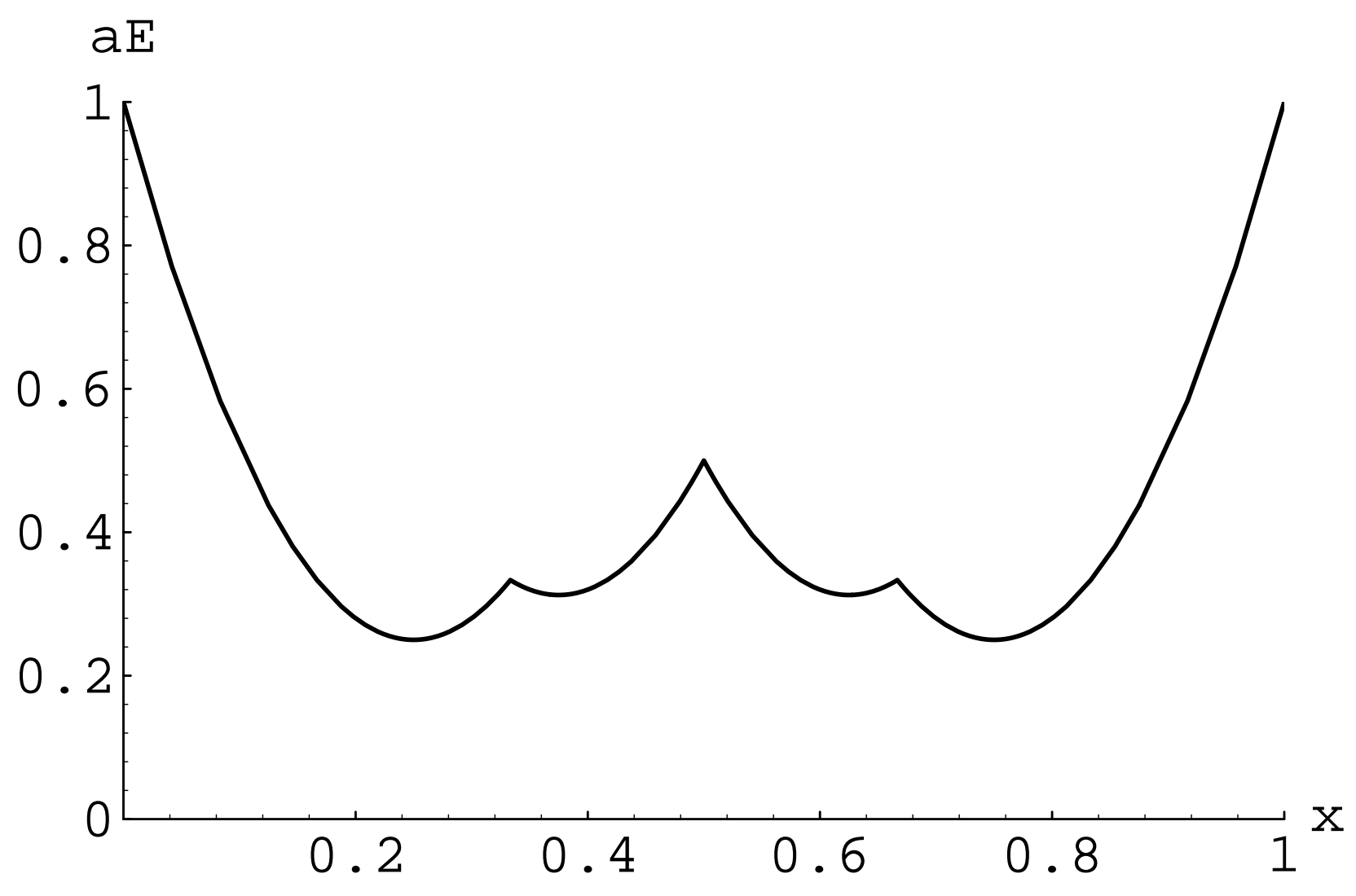


This figure "fig1-1.png" is available in "png" format from: http://arxiv.org/ps/hep-ph/9411375v2 
This figure "fig1-2.png" is available in "png" format from: http://arxiv.org/ps/hep-ph/9411375v2 
(6) 